\title{
CartoMerge using SoundStar Catheter and Time Force Integral-Based Ablation for Atrial Fibrillation
}

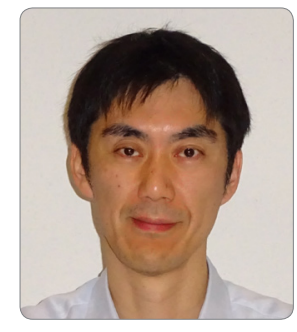

Koichi Inoue, MD, PhD

Cardiovascular Center, Sakurabashi Watanabe Hospital, Osaka, Japan
Received: December 21, 2016

Revision Received: February 14, 2017

Accepted: February 14, 2017

Correspondence: Cardiovascular Center, Sakurabashi Watanabe Hospital, 2-4-32 Umeda, Kita-ku, Osaka 530-0001, Japan.

E-mail: koichi@inoue.name

Copyright $(2017$ The Official Journal of Korean Heart Rhythm Society Editorial Board \& MMK Co., Ltd.

\begin{abstract}
Catheter ablation for atrial fibrillation is based on pulmonary vein (PV) isolation, but this procedure is thought to be demanding. The visualization of 3-dimensional information that is provided by CartoMerge $^{\circledR}$ (BioSense Webster Inc., Diamond Bar, CA, USA) makes the ablation procedure easier. The SoundStar ${ }^{\circledR}$ catheter (BioSense Webster Inc., Diamond Bar, CA, USA)became available in Korea in September 2016. CartoMerge ${ }^{\circledR}$ using a SoundStar ${ }^{\circledR}$ catheter, which we termed as SoundMerge, is a simple way to obtain good CartoMerge ${ }^{\circledR}$ results. In addition, information on catheter stability and ablation intensity at each site are provided by a new ablation annotation system (CARTO ${ }^{\circledR} 3$ System, VisiTag ${ }^{\text {TM }}$ Module [BioSense Webster Inc., Diamond Bar, CA, USA]), which would be helpful for accomplishing durable PV isolation. In this article, we introduce the methodology of SoundMerge and the setting of the VisiTag ${ }^{\text {TM }}$ module that we are performing currently. Effective practical use of these new technologies would improve the quality of ablation procedures.
\end{abstract}

Key Words: - Atrial fibrillation -Cather ablation

\section{Background}

Catheter ablation is a treatment that can possibly cure atrial fibrillation, and has benefitted many patients having this arrhythmia. The strategy for this ablation technique is based on pulmonary vein $(\mathrm{PV})$ isolation, but the outcome and procedure time differ between institutions and operators because of technical difficulties.

The main reasons for these difficulties are the complicated morphology of the left atrium (LA) and PV, individual differences in their anatomy, and difficulty in fixing the 3-dimensional location of the points that were ablated and those to be ablated. Three-dimensional mapping systems, such as CARTO $^{\circ}$ (BioSense Webster Inc., Diamond Bar, CA, USA), provide useful information to the operator by visualizing 3 dimensional locational information of the position. CartoMerge is a function used to overlay computed tomography (CT), or magnetic resonance imaging (MRI) images, on to a $\mathrm{CARTO}^{\circ}$ image. The visualization of 3-dimensional information makes the ablation procedure easier; therefore, improved ablation performance is 
expected. There are several methods for performing CartoMerge ${ }^{\circ}$ We proposed that CartoMerge using a SoundStar ${ }^{\circ}$ catheter, which we termed as SoundMerge herein, is the simplest way to obtain good CartoMerge results.

Navistar ${ }^{\circ}$ ThermoCool ${ }^{\circ}$ (BioSense Webster Inc., Diamond Bar, CA, USA) contact force (CF) enabled CF monitoring of the ablation catheter, which may contribute to achieving more durable lesions. However, a recent randomized, multicenter study indicated that CF data availability was not associated with improved 1-year success rates. ${ }^{1}$ This is possibly due to a lack of information on catheter stability and subsequent heterogeneous ablation intensity. An ablation annotation system $\left(\mathrm{CARTO}^{\circ} 3\right.$ System, VisiTag ${ }^{\text {Tu }}$ Module) permits the automated annotation of radiofrequency (RF) ablation, based on objective, predefined parameters. It provides crucial information on catheter stability and ablation intensity at each site, and may be useful for accomplishing durable $\mathrm{PV}$ isolation.

Here, we would like to introduce the method of SoundMerge and the setting of the VisiTag ${ }^{\text {max }}$ module that we are performing currently.

\section{SoundStar ${ }^{\circledR}$ Catheter}

The SoundStar ${ }^{\circ}$ catheter became available in April 2012 in Japan, and then in September 2016 in Korea. The SoundStar ${ }^{\circ}$ catheter is an ultrasound catheter dedicated for use inside the heart chamber. At the tip of the catheter, a magnetic sensor and an ultrasonic probe are mounted. The location and direction of this magnetic sensor are recognized by the $\mathrm{CARTO}^{\circ} 3$ system, and like the Navistar ${ }^{\circ}$ catheter, the ultrasonic beam orientation is recorded in real time by the $\mathrm{CARTO}^{\circ} 3$ system. Therefore, it is possible to create a 3-dimensional anatomical map (Shell) by tracing the edge of LA in the acquired ultrasound images.

\section{Procedure of SoundMerge}

SoundMerge consists of two steps; landmark registration with a single point landmark, and surface registration using the Shell created on the posterior wall and roof of the LA and PVs. Specific procedures are detailed below.

\section{Creating a landmark (Figure 1a).}

To perform landmark registration, at least one landmark must be set at an easily recognizable anatomical position found on both the ultrasound and CT image incorporated in the $\mathrm{CARTO}^{\circ} 3$ system. The rear side of the right PV carina is the easiest site to landmark.

2. Creation of contour on the left atrial wall (Figure 1b).

When the SoundStar ${ }^{\circ}$ catheter is placed in the right atrium (RA), the left atrial posterior wall is drawn when the ultrasound is directed from approximately 4 o'clock (left PV side) to 7 o'clock (right PV side). Subsequently, the left atrial posterior wall is traced on to the ultrasound image captured by the CARTO 3 system. This line is called contour, and it is a collection of points with location information. Using these points, we create a 3-dimensional geometry called Shell.

\section{Landmark and surface registration (Figure 1c).}

After landmark registration with one landmark surface registration is performed using Shell, the $\mathrm{CARTO}^{\circ} 3$ system automatically shifts the CT image of the LA so that the average distance between the CT image and each point on the contour is minimalized. Each step ends with just one click.

\section{Deletion of incorrect contours.}

There is a function to display the distance, in millimeters, between each point and the CT image surface. Anomalously large distances are deleted. Points can be added with the NAVISTAR ${ }^{\circ}$ catheter, if necessary.

\section{Adding points with a Navistar catheter.}

If clear echo images cannot be obtained, points can be added using a Navistar catheter. The catheter should be manipulated into contact with the wall of LA or PV at the problematic sites, and points can be added to the Shell, using the electrocardiogram (ECG)-gated mode. The details are shown below.

6. Verifying that MERGE was performed correctly.

A lasso catheter can be inserted into each PV. If it is displayed in each PV on the CARTO 3 system, then MERGE is accurate. The time taken for this series of procedures is approximately 5 minutes.

As mentioned above, we can display the distance between the point used for MERGE and the CT image. The average distance between the CT image and points on the contours was 1.4 \pm 1.0 $\mathrm{mm}$ in 44 successive cases at our early stage. 
A

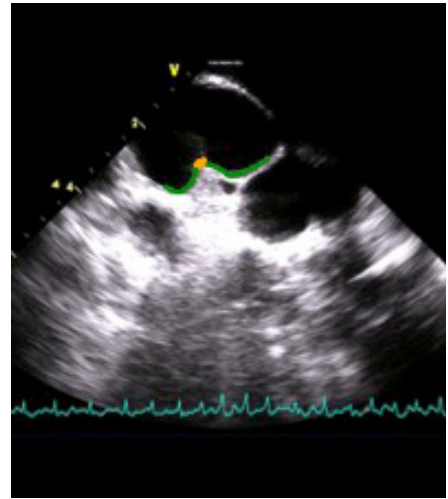

B

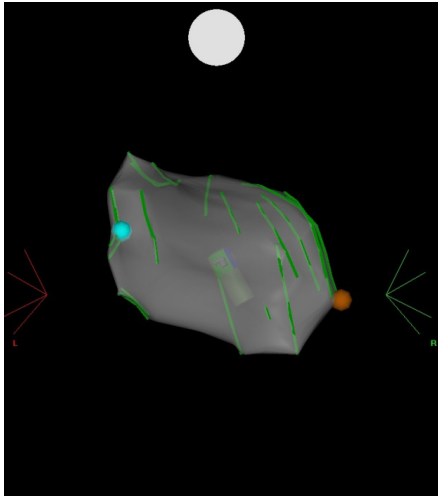

C

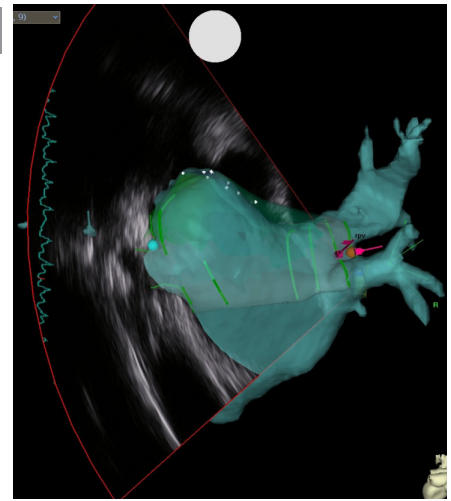

Figure 1. Example of SoundMerge.

A. The orange point indicates a landmark on the CARTO ${ }^{\circledR}$ image. The posterior carina of the right pulmonary vein, which can be visualized and easily identified in most cases, is the best option for the landmark. Green lines indicate contours drawn by CARTO ${ }^{\circledR}$ operators.

B. Shell of the left atrium. Green lines indicate contours.

C. After landmark registration and surface registration. Ultrasound image is also shown here.

A

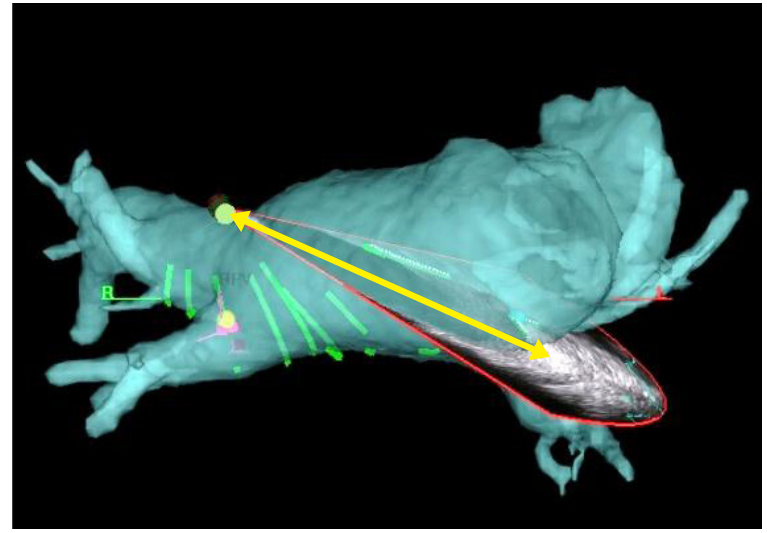

B

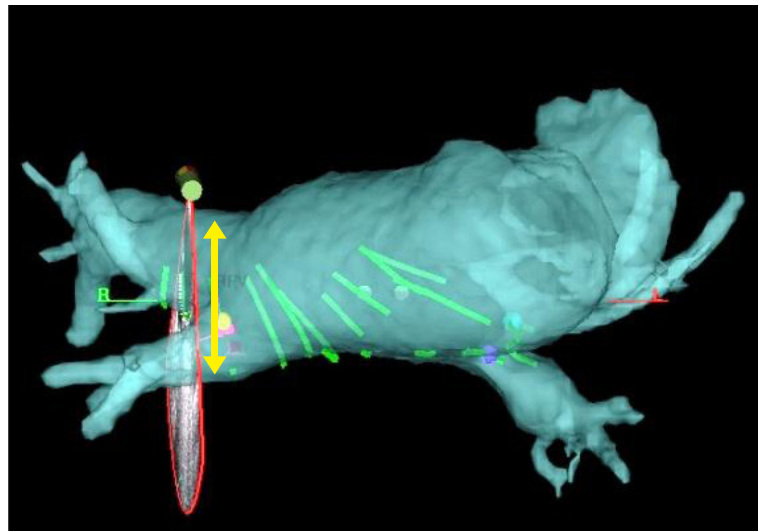

Figure 2. Comparison of the distance between the probe and the left atrial posterior wall.

The yellow arrow indicates the distance from the SoundStar ${ }^{\circledR}$ catheter to the atrial wall. The distance from the left side of the left atrium to the probe $(\mathrm{A})$ is longer than the distance from the right side of the left atrium (B), and the angle with the rear wall is steeper in left side.

\section{Tips for SoundMerge quality}

There are some crucial tips to obtaining high-quality SoundMerge.

1. Trace only the posterior wall of LA and PV.

As the anterior wall, lateral wall, septum, and inferior wall of LA easily expand and contract, the size and shape will change because of a slight difference in conditions. Conversely, because PVs are fixed to the hilar, and the posterior wall has a spine behind it, they cannot expand as effortlessly as the septum, anterior wall, and lateral wall. Therefore, the degree of change because of the conditions at the posterior wall and PVs is relatively small. It is strongly recommended that contour be created by tracing only the posterior wall and the roof close to the posterior wall of the LA and PVs.

2. Create CT images, as well as contours, at end systole.

The shape and size of the LA vary depending on the cardiac cycle. For this reason, the CT image and SOUNDMAP Shell must be created in the same phase of the cardiac cycle. We recommend end systole, which is when the size of the LA is maximized. CT imaging should be taken at $40-50 \%$ of the cardiac cycle, ${ }^{2}$ and contour should be drawn at the end of the $\mathrm{T}$ 
wave.

3. Create a SOUNDMAP in accordance to the respiratory cycle.

The position of the heart moves several centimeters during breathing. As the diaphragm goes down during inspiration, the lungs are stretched vertically, and the PV morphology changes. ${ }^{3}$ For these reasons, SOUNDMAP must be created and CT image must be taken during the expiratory phase.

4. Make a SOUNDMAP before insertion of transseptal sheaths and catheters into the LA.

The transseptal sheaths and catheters, indwelled in the LA, obstruct the echo beam making it difficult to visualize the LA. Therefore, we recommend MERGE before transseptal puncture. 5. Do not completely rely on the SoundStar image, particularly in the left side of the LA.

The left side of the LA is far from the SoundStar ${ }^{\circ}$ catheter in the right atrium (Figure 2). Due to attenuation of the echo, it is often difficult to obtain a good image compared with the right side, where the distance is minimal. In addition, the angle between the posterior wall and the echo beam is steep on the left side, thus it is difficult to obtain a good image, and the edges are easily blurred.

In case of difficulty in obtaining a good image of the LA because of attenuation of the echo, the LA should be depicted through the foramen ovale, which has a thin wall. Another option is to manipulate the SoundStar ${ }^{\circ}$ catheter slightly, so that it contacts the septum, where the distance from the left side of the LA is minimized.

6. Add points using a Navistar ${ }^{\circ}$ catheter.

Nevertheless, the left side LA may not be well rendered. In such a case, you can create additional points using a Navistar ${ }^{\circ}$ catheter as
A

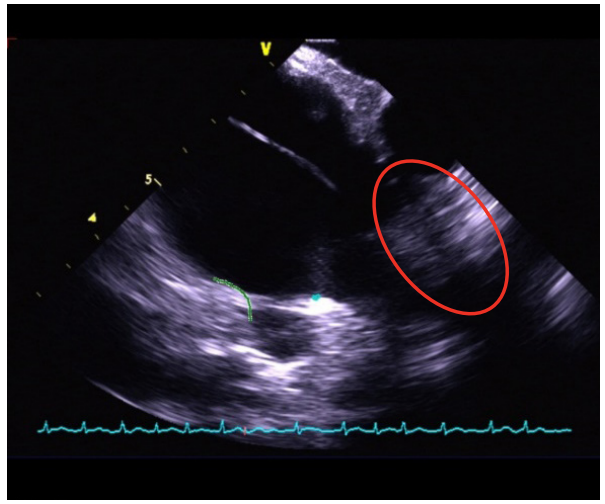

C

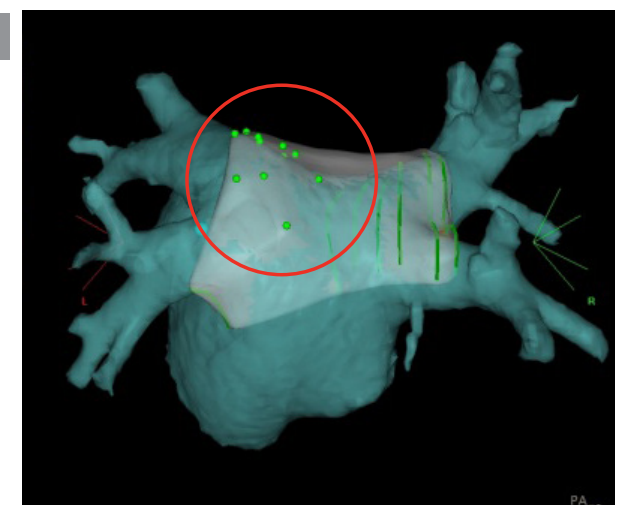

B

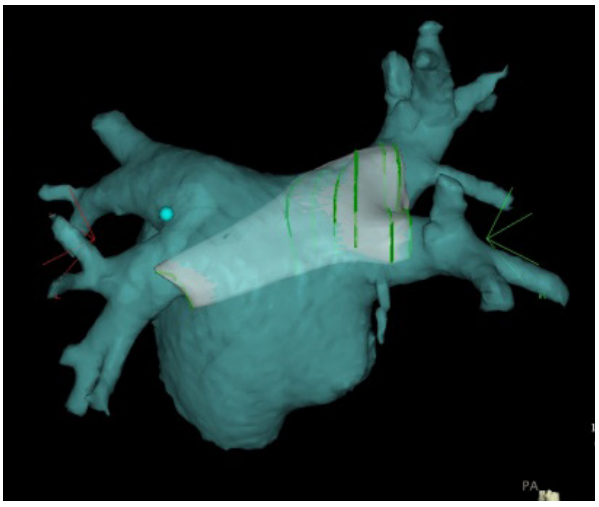

D

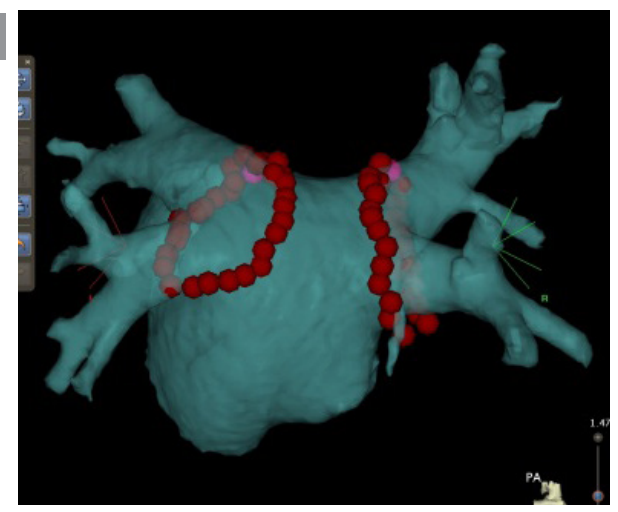

Figure 3. Correcting a poor image of the left side of the left atrium. In this case, the image of the left atrium is vague (indicated by red circle in Figure A). It was impossible to draw contours of this position (B), and the accuracy of CartoMerge ${ }^{\circledR}$ at that site was poor. Therefore, we added position information on the posterior wall of the left atrium and the roof of the pulmonary vein, which could not be seen with CARTOSOUND ${ }^{\circledR}$, using a Navistar ${ }^{\circledR}$ catheter (the green dots in Figure C). By doing this, accurate MERGE could be obtained successfully (D). 
A

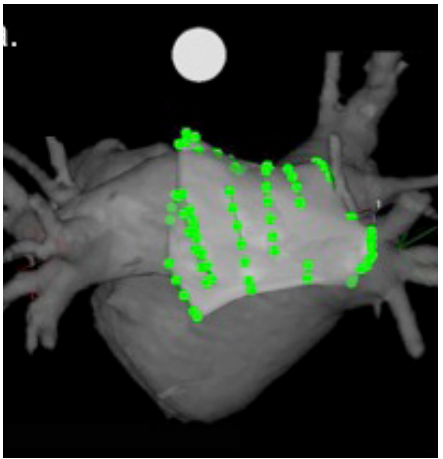

B

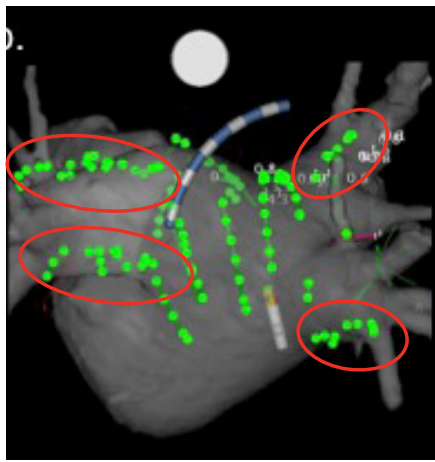

C

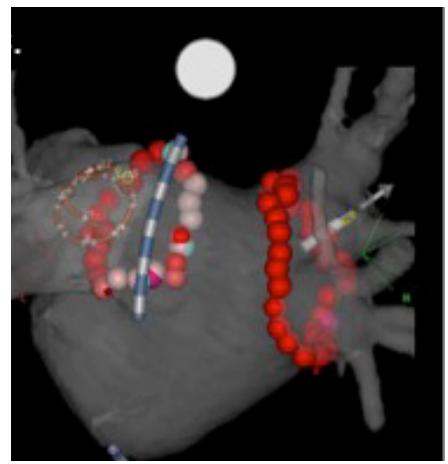

Figure 4. Addition of locational information using Navistar ${ }^{\circledR}$.

Currently, we routinely add points linearly using a Navistar ${ }^{\circledR}$ catheter at the roof of the left and right superior pulmonary veins and at the bottom of the left and right lower pulmonary veins.

a. Points and Shells obtained with a SoundStar ${ }^{\circledR}$ catheter.

b. Points indicated by red circles were obtained by a Navistar ${ }^{\circledR}$ catheter. Using these points for CartoMerge ${ }^{\circledR}$, the left atrium image was rotated clockwise. c. Ablated points tagged with the VisiTag ${ }^{\mathrm{TM}}$ module were accurate on the CT image of the left atrium, indicating that this CartoMerge ${ }^{\circledR}$ was accurate. CT, computed tomography
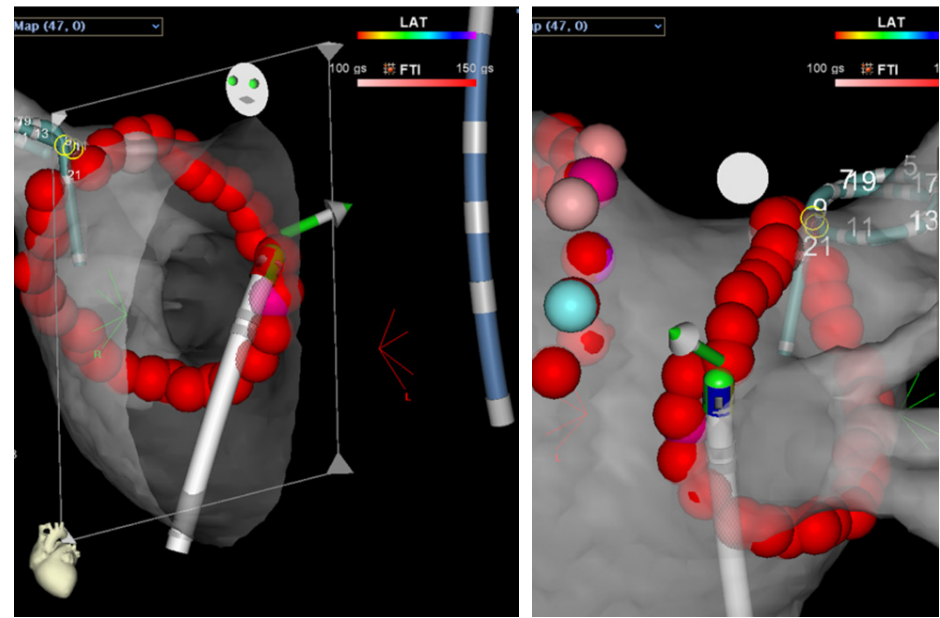

Figure 5. VisiTag ${ }^{\mathrm{TM}}$ module guided pulmonary vein isolation.

Ablated points are displayed in tags with a diameter of $6 \mathrm{~mm}$. The color of the tag turns red when FTI reaches 150 or more. We aim to ablate so that the ipsilateral pulmonary veins are linearly surrounded by red tags without gaps. Left panel; inner view, right panel; outer view.

FTI, force time integral; LAT, local activation time

described above.

The $\mathrm{CARTO}^{\circ} 3$ system has a stable mode, and an ECGsynchronized gated mode. The points must be recorded in gated mode linearly in the expiratory phase. An example is shown in Figure 3. Because we could not visualize the left upper PV, CT images were down to the left shoulder even when CartoMerge was performed. By using NAVISTAR ${ }^{\circ}$ to create contour on the ceiling of the left superior PV, we could obtain good MERGE.

Recently, to obtain an improved CartoMerge ${ }^{\circledR}$, we routinely add points linearly using Navistar ${ }^{\circ}$ at the roof of the left and right superior PVs, and at the bottom of the left and right lower PVs. Performing this simple and easy step will greatly improve the accuracy of CartoMerge ${ }^{\circ}$ (Figure 4 ). 


\section{Annotation using VisiTag ${ }^{\mathrm{TM}}$}

Another recent noteworthy advance in technology for catheter ablation is the measurement of catheter stability. A new ablation annotation system (CARTO 3 System, VisiTag ${ }^{\text {mex }}$ Module) permitted the automated annotation of RF ablation, based on objective parameters of catheter stability. Parameters of the VisiTag $^{\text {tw }}$ module were set as follows; ${ }^{1}$ catheter stability range of motion $\leq 1.5 \mathrm{~mm},{ }^{2}$ catheter stability duration $>5 \mathrm{~s}$, and $\mathrm{d}^{3}$ force divided by time $>5 \mathrm{~g}$ and $>25 \%$, respectively. Tag size was $6 \mathrm{~mm}$ in diameter. We ablated each lesion for more than $20 \mathrm{~s}$, and the force time integral (FTI) of each lesion is $>150$ gs. To find optimal ablation intensity in this setting, we retrospectively analyzed data from 5544 ablated lesions, during 77 consecutive procedures with initial pulmonary vein isolation (PVI), using CF monitoring in our institute. We found that FTI $<150$ gs resulted in a significant risk for residual conduction gaps. We intended to ablate densely, so that tags overlapped with each other, and thus a circular line of red tags surrounded the ipsilateral PVs without gaps (Figure 5).

\section{Conclusion}

The CARTO 3 system is a user friendly 3-dimensional mapping system, which obtains accurate MERGE using SoundMerge, and can annotate ablation lesions, thereby monitoring catheter stability and contact force. This system improves the ablation quality achieved by young physicians, reducing the difference in the results obtained between them and highly skilled physicians. It is thought that it contributes to improved performance and safety during atrial fibrillation ablation.

\section{References}

1) Ullah W, McLean A, Tayebjee MH, Gupta D, Ginks MR, Haywood GA, O'Neill M, Lambiase PD, Earley MJ, Schilling RJ; UK Multicentre Trials Group.W Ullah. Randomized trial comparing pulmonary vein isolation using the smarttouch catheter with or without real-time contact force data. Heart Rhythm. 2016;13:1761-1767.

2) Patel AR, Fatemi O, Norton PT, West JJ, Helms AS, Kramer CM, Ferguson JD. Cardiac cycle-dependent left atrial dynamics: Implications for catheter ablation of atrial fibrillation. Heart Rhythm. 2008;5:787-793.

3) Ector J, De Buck S, Loeckx D, Coudyzer W, Maes F, Dymarkowski S, Bogaert J, Heidbüchel H. Changes in left atrial anatomy due to respiration: impact on three-dimensional image integration during atrial fibrillation ablation. J Cardiovasc Electrophysiol. 2008;19:828-834. 